\title{
Contouring by electronic speckle pattern interferometry employing dual beam illumination
}

\author{
Charles Joenathan, Berthold Pfister, and H. J. Tiziani
}

\begin{abstract}
In this paper we extend and study the method for generating contours of diffuse objects employing a dual beam illumination coupled with electronic speckle pattern interferometry. The sensitivity and the orientation of the contour planes are analyzed. A novel method for tilting the planes of contours and experimental results incorporating phase shifting and fringe analysis are also presented. The theoretical and the experimental results show good agreement. Key words: Interferometry, speckles, speckle interferometry, ESPI
\end{abstract}

\section{Introduction}

Generation of contours of constant depth of an object provides data for 3-D shape analysis and has numerous uses such as data for numerically controlled machines, biomedical research, and automatic inspection, to name a few. Holographic methods for measuring surface shapes are based on two-wavelength techniques, ${ }^{1}$ altering the refractive index of the medium surrounding the object, ${ }^{2}$ sandwich holographic interferometry, ${ }^{3}$ and by displacing the object and the illuminating beam. ${ }^{4}$ Electronic speckle pattern interferometry (ESPI) an outgrowth of image plane holography, has received some of the direct implementation of the holographic methods. The advantage of employing ESPI is that intermediate photographic processing is eliminated. Added to it, is the possibility of including a host computer to analyze the fringes by a phase shift method. Some of the well known ESPI techniques for contouring and shape analysis have already been discussed in various books in the literature..$^{5,6}$

Recently some new methods for contouring have been reported in ESPI to overcome some of the drawbacks suffered by the already existing optical methods. ${ }^{7,8}$ Studies have been recently carried out with a dual beam interferometer for generating contours of

\footnotetext{
When this work was done all authors were with University of Stuttgart, Institute for Technical Optics, D-7000 Stuttgart 80, Federal Republic of Germany; C. Joenathan is now with Rose-Hulman Institute of Technology, Department of Applied Optics Studies, 5500 Wabash Avenue, Terre Haute, Indiana 47803.

Received 24 July 1989.

0003-6935/99/131905-07\$02.00/0.

(C) 1990 Optical Society of America.
}

an object. ${ }^{8}$ The interferometer has the advantage that the imaging system does not have to resolve the fine grating structure and can therefore be easily adapted to ESPI. These interferometers have been used frequently for the measurement of in-plane displacements $^{9}$ and out-of-plane displacements. ${ }^{10}$

In this paper we present detailed studies made of the sensitivity and the orientation of the contour planes. We also show that many conditions must be met to obtain the equation for fringe formation. Moreover, a method for inclining the contour planes is reported. The interferograms obtained by this method are analyzed by phase shifting techniques. Three phase shifted interferograms are recorded sequentially for evaluating the phases. The results with phase shifting and fringe analysis are also presented.

\section{Experimental Arrangement and Theory}

Figure 1 shows the schematic of the experimental arrangement of a dual beam interferometer. The laser beam is split into two illuminating beams $A$ and $B$ of equal amplitudes via a beam splitter $B S$. The beams upon reflection from mirrors are expanded and collimated. To introduce a phase shift one of the mirrors is attached to a piezoelectric translator (PZT). The two beams with an included angle illuminate the object to be contoured. ATV camera images the object which is digitized by a host computer and displayed on a monitor. Two exposures are made with the object tilted between the exposures, and the subtracted speckle pattern is displayed on the monitor. In the following discussion we assume that the tilt and rotation of the object do not displace the speckle; only a phase change occurs.

Let $A_{1}$ and $A_{2}$ be the complex amplitudes of the waves due to the illuminating beams $A$ and $B$ respectively. The fringes are obtained by subtracting the 


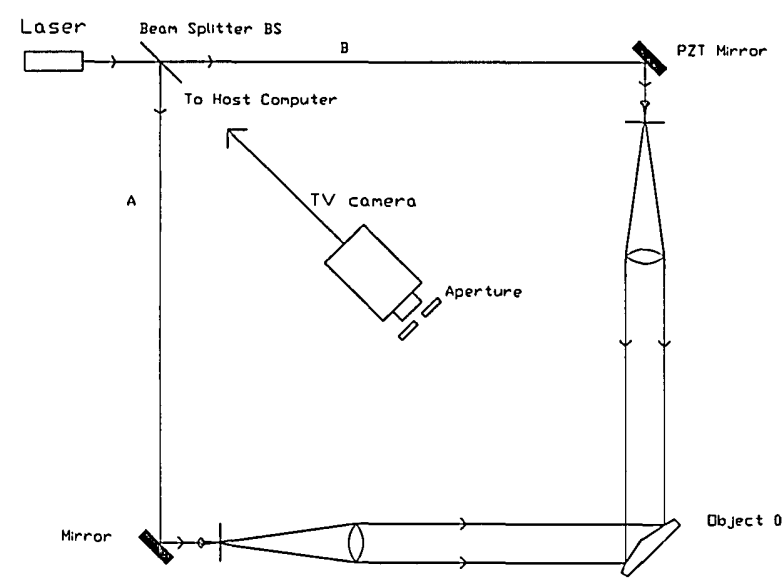

Fig. 1. Schematic of the experimental setup of a dual beam interferometer for contouring applications.

intensity record before and after tilt of the object. The difference is then squared or the modulus is taken, thereby giving,

$$
I_{1}-I_{2}=\left|4 A_{1} A_{2} \sin [\phi(x, y)-\Delta / 2] \sin (\Delta / 2)\right|,
$$

where $\phi(x, y)$ is the random phase of the speckle pattern and $\Delta$ is the phase shift due to the object deformation. The output of the camera will have voltages proportional to the intensities, i.e., $V_{1} \propto I_{1}$ and $V_{2} \propto I_{2}$. The difference between the two voltages (proportional to the difference between the intensities) is displayed on the TV monitor.

\section{Sensitivity}

The schematic geometry of the illumination and observation angle is shown in Fig. 2. The collimated beams $A$ and $B$ impinge on the object at angles $\theta_{1}$ and $\theta_{2}$ along the $x z$-plane and $\phi_{1}$ and $\phi_{2}$ along the $y z$-plane. The direction of observation is at an angle $\theta_{3}$ to the $z$ axis. The unit vectors for beam $A$, and $B$, and the observation direction are $\hat{k}_{1}, \hat{k}_{2}$ and $\hat{k}_{3}$ respectively. The small tilt of the object introduces speckle displacements and the phase variation can be expressed as

$$
\Delta=\frac{2 \pi}{\lambda}\left(\hat{k}_{1}-\hat{k}_{2}\right) \cdot \mathbf{L},
$$

where $\mathbf{L}=u \hat{i}+v \hat{j}+w \hat{k}$. Here we have assumed collimated beam illumination to maintain $\hat{k}_{1}$ and $\hat{k}_{2}$ constant throughout the object surface. When the illumination beam is diverging to accommodate large objects, the divergence of the beam should be made large enough such that the illumination vectors can be assumed to be constant.

Substituting for $\hat{k}, \hat{k}_{2}$, and $\mathrm{L}$, it is observed that all the three components of displacement contribute to the pathlength change. If $\left|\theta_{1}\right| \simeq\left|\theta_{2}\right|$ and $\left|\phi_{1}\right| \simeq\left|\phi_{2}\right|$ (If the object surface slope variation is small) the $w$-component is reduced. The $w$-component that arises due to the tilt of the object can be expressed as

$$
w=\left(r^{2}-h^{2}\right)^{1 / 2} \phi_{t},
$$

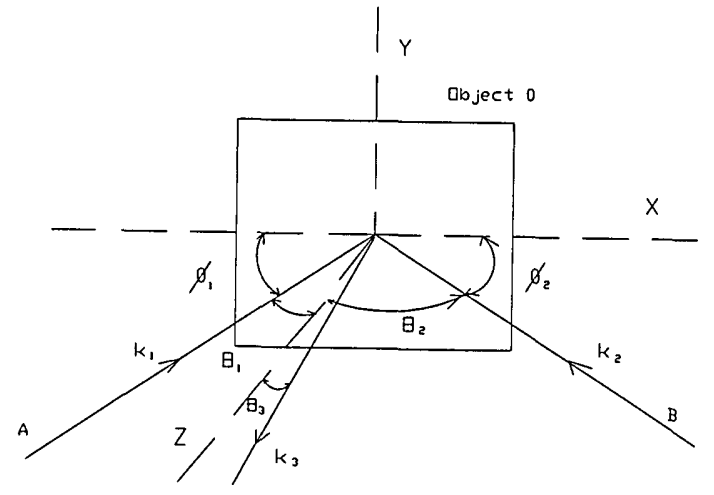

Fig. 2. Geometry of the illumination and observation directions.

where $r$ is the radial coordinate of a point on the object and the origin of the coordinate is the center of the tilt axis, $h$ is the distance of a point of the object with respect to the tilt plane, and $\phi_{t}$ is the tilt of the object. $w$ is maximum at the periphery of the object. If $r$ is set large, i.e., by shifting the tilt axis far behind the object, $r \sim h$ throughout the object surface, thereby eliminating the effect of the $w$-component of displacement irrespective of slope of the object and Eq. (2) reduces to

$$
\begin{aligned}
\Delta= & \frac{2 \pi}{\lambda}\left[\left(\sin \theta_{1} \cos \phi_{1}+\sin \theta_{2} \cos \phi_{2}\right) u\right. \\
& \left.+\left(\sin \phi_{1} \cos \theta_{1}+\sin \phi_{2} \cos \theta_{2}\right) v\right]
\end{aligned}
$$

Let us assume that the object is arbitrarily tilted about the $x y$-plane. In this particular illumination geometry the components of inplane displacement are introduced. If the plane of tilt is at an angle $\beta$ to the $x$ axis, we can write the following equations,

$$
\begin{gathered}
u=h \phi_{t} \cos \beta \\
v=h \phi_{t} \sin \beta .
\end{gathered}
$$

The approximation in Eqs. (5a) and (5b) holds only when $h \gg u$ and $v$ respectively. The equation for the pathlength becomes

$$
\begin{aligned}
\Delta= & \frac{2 \pi}{\lambda} h \phi_{t}\left[\left(\sin \theta_{1} \cos \phi_{1}+\sin \phi_{2} \cos \phi_{2}\right) \cos \beta\right. \\
& \left.+\left(\sin \phi_{1} \cos \theta_{1}+\sin \phi_{2} \cos \theta_{2}\right) \sin \beta\right] .
\end{aligned}
$$

The sensitivity depends on the angle of illumination, angle of tilt $\phi_{t}$, and the orientation of the tilt $\beta$. However, the contribution from the $v$ component, for an illumination lying near to the $x z$ plane is very small and can be eliminated by adjusting $\beta$ to be very small.

Making $\left|\theta_{1}\right| \simeq\left|\theta_{2}\right|$ for large illumination angles, the equation for the fringes of constant depth reduces to a simple form as derived in Ref. 8 and is

$$
\Delta h=\frac{\lambda}{2 \phi_{t} \sin \theta} \text {. }
$$

The contour fringes are dependent on two factors: a) the tilt $\phi_{t}$ and b) the orientation of the object. The planes of contour elevation as seen from Eq. (7) lie parallel to the $x y$-plane, irrespective of the direction of 
observation. The contour fringes are also independent of the origin of the tilt axis. For an observation direction not along the $z$-axis, the perspective view of the object changes, and not the contour planes. In the following section we introduce a method to tilt the contour planes about the $x y$-plane. Accuracy of the method can be seen to depend mainly on the accuracy of the tilt angle measurement. The error introduced in the depth contour is determined to be

$$
\Delta h_{e}=\frac{\lambda \Delta \phi_{t}}{2 \phi_{t}^{2} \sin \theta},
$$

where $\Delta \phi_{t}$ is the error in measuring $\phi_{t}$.

\section{Tilt of the Contour Planes}

In general a different perspective view of the object is sometimes necessary to obtain a better picture of the 3-D shape of the object. In this section we introduce a simple method to incline the contour planes with respect to the $x y$-plane. The orientation of the contour planes is changed by introducing a small rotation to the object along with the tilt. For simplicity, assuming that the object is rotated by $\phi_{r}$ about its center, the new coordinates of an object point after rotation can be expressed as

$$
\begin{aligned}
& x^{\prime}=x \cos \phi_{r}-y \sin \phi_{r} \\
& y^{\prime}=x \sin \phi_{r}+y \cos \phi_{r} .
\end{aligned}
$$

The in-plane components of motion can be written as

$$
\begin{aligned}
& u_{r}=x-x^{\prime}=x\left(1-\cos \phi_{r}\right)+y \sin \phi_{r} \\
& v_{r}=y-y^{\prime}=x \sin \phi_{r}-y\left(1-\cos \phi_{r}\right) .
\end{aligned}
$$

The combined in-plane components of motion due to tilt and rotation either increases or decreases, depending upon the coordinate of the point under consideration, and, as we have chosen the rotation to be about the center of the object the effective $u_{r}$ and $v_{r}$ components due to rotation and tilt of the object are

$$
\begin{aligned}
& u_{T}=u_{t} \pm u_{r} \\
& v_{T}=v_{t} \pm v_{r},
\end{aligned}
$$

where $u_{t}$ and $v_{t}$ are the in-plane displacement due to tilt of the object. Substituting for $u_{t}, u_{r}, v_{t}$ and $v_{r}$, Eqs. (11a) and (11b) become

$$
\begin{aligned}
& u_{T}=h \phi_{t} \cos \beta+\left\{x\left(1-\cos \phi_{r}\right)+y \sin \phi_{r}\right\} \\
& v_{T}=h \phi_{t} \sin \beta+\left\{x \sin \phi_{r}+y\left(1-\cos \phi_{r}\right)\right\} .
\end{aligned}
$$

The rotation of the object increases the in-plane motion for positive $x$ - and $y$-coordinates, and reduces it for negative $x$ - and $y$-coordinates if $\phi_{r}$ is positive. Since $\phi_{r}$ and $\beta$ are small the equation for the phase can be written as

$$
\Delta=\frac{4 \pi}{\lambda}\left[h \phi_{t}+y \phi_{r}\right] \sin \theta .
$$

The fringes of constant elevation become
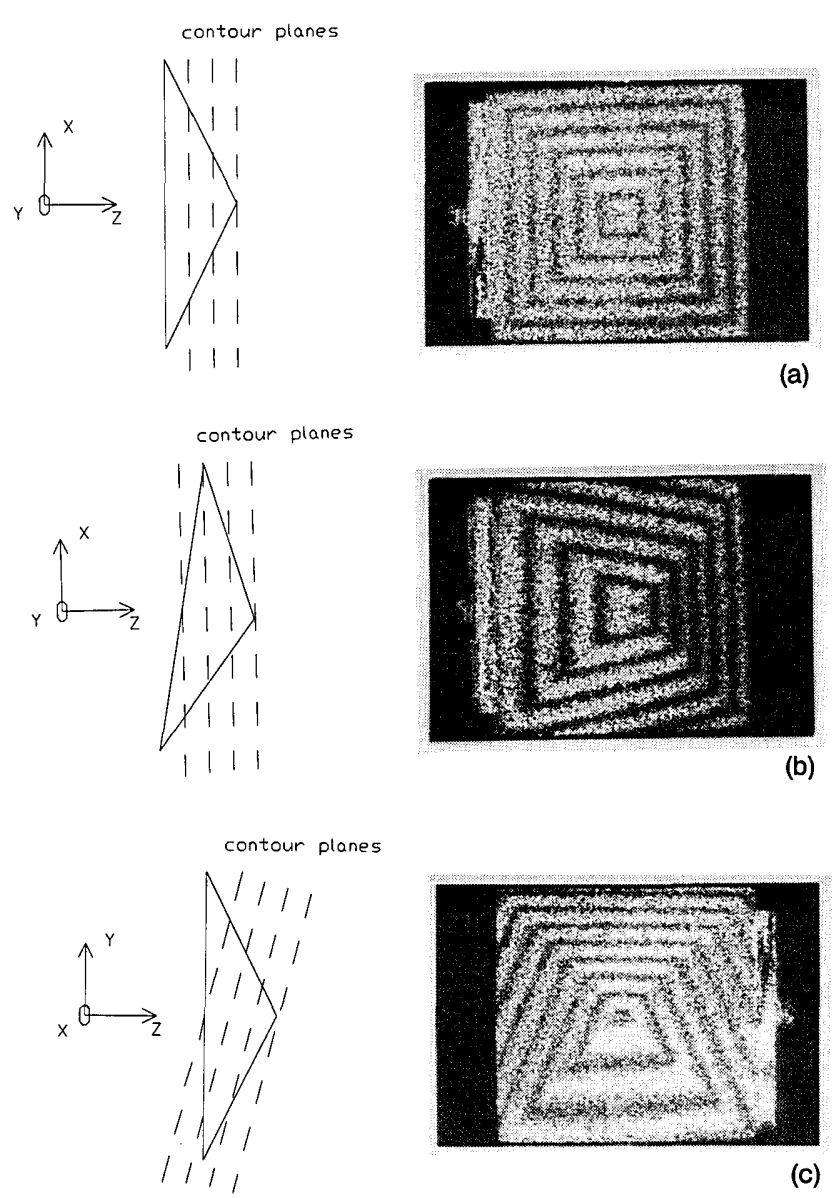

(c)

Fig. 3. Photographs of the contour fringes of constant depth for a pyramid. a) The contour planes are perpendicular to the $Z$-axis: $\beta$ $=0, \phi_{t}=0.2 \mathrm{mrad}, \theta=45^{\circ}$, and $\Delta h=2.23 \mathrm{~mm}$. b) The object is tilted by $10^{\circ}$ about the $Y$-axis: $\beta=0, \phi_{t}=0.15 \mathrm{mrad}, \theta=45^{\circ}$, and $\Delta h=$ $2.98 \mathrm{~mm}$. c) The contour planes are inclined about the $X$-axis. This is realized by rotating the object along with the tilt: $\phi_{r}=0.05$ $\operatorname{mrad}, \theta^{\circ}=14^{\circ}, \phi_{t}=0.2 \mathrm{mrad}, \theta=45^{\circ}$, and $\Delta h=2.23 \mathrm{~mm}$.

$$
\Delta h=\frac{\lambda}{2 \phi_{t} \sin \theta}-\frac{\left(y_{1}-y_{2}\right) \phi_{r}}{2 \phi_{t}},
$$

where $y_{1}$ and $y_{2}$ are the $y$-coordinate between two fringes or, in other words, it is the fringe width. The orientation of the planes of contour elevation with respect to the $x y$-plane is

$$
\theta_{0}=\tan ^{-1}\left[\phi_{r} / \phi_{t}\right]
$$

The contour planes can be tilted about the positive and negative values of $\theta_{0}$ by the choice of direction of rotation of the object. As $\phi_{r}$ is increased, the pathlength variation due to the height variation becomes small and the fringes are mainly due to the rotation of the object. It is clear that for a geometry of illumination considered here, the contour planes can be tilted only about the $x z$-plane.

Following Ref. 8, the corresponding tilt and rotation angles for the fringes to vanish can be shown to be

$$
\left(h \phi_{t}+y_{\max } \phi_{r}\right)=2.4 \lambda p /(2 M a),
$$

where $p$ is the distance between the lens and the image plane, $M$ is the magnification, and $a$ is the diameter of 


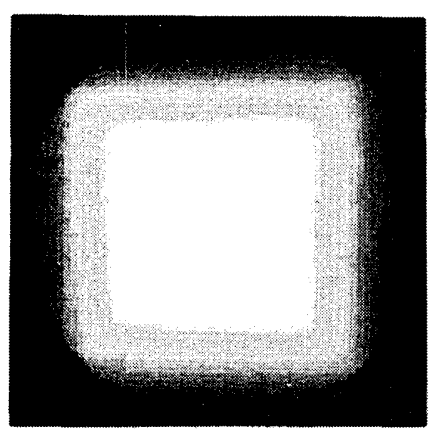

a

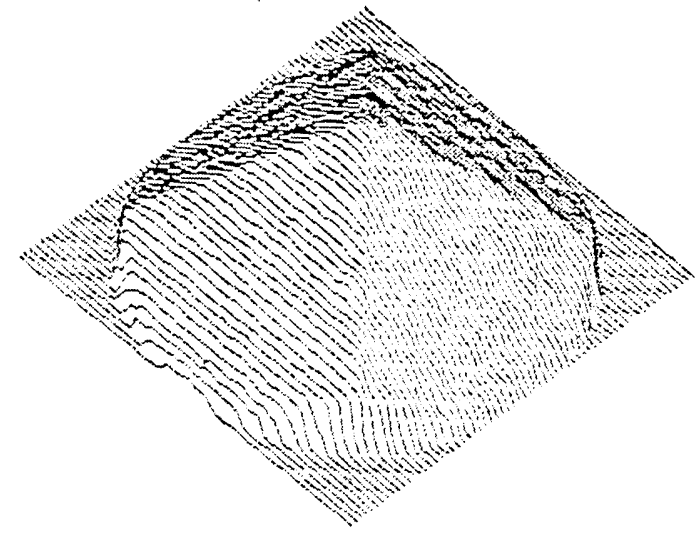

b

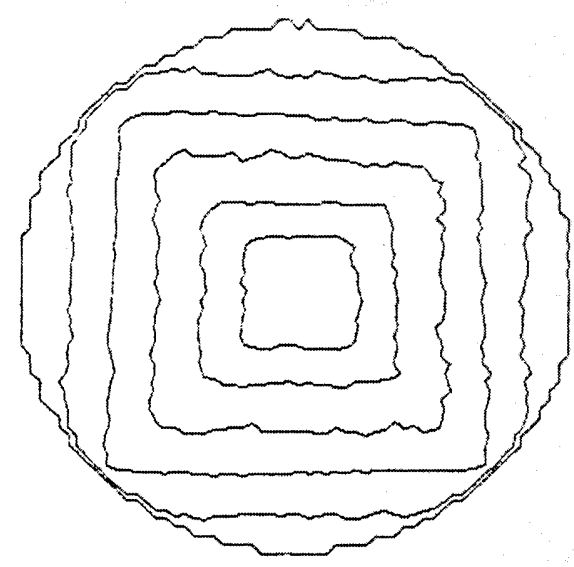

$\mathbf{c}$

Fig. 4. Results obtained with the three interferogram method for the pyramid with the interferogram shown in Fig. 3(a). a) Phase maps. b) 3-D plot. c) Plot of the contour maps.

the circular aperture and $y_{\max }$ is the maximum of the $y$ coordinate of the object that is contoured. Since the coherence factor varies as a Bessel function, the visibility of the fringes starts rising beyond the extreme values of tilt and rotation angles.

\section{Phase Shifting Dual Beam Speckle Interferometry}

To obtain quantitative data from the interferograms, the phase of one of the illuminating beams in the speckle interferometer is shifted with respect to the other in steps. The phase maps are calculated from the phase shifted intensity data taken before and after the tilt of the object. The phases of the difference between the speckle patterns are evaluated by taking three frames of intensity data, while shifting the phase of one of the beams.

Three frames of intensity data are recorded and they are

$$
\begin{aligned}
& I_{1}=I_{0}[1+\gamma \cos (\Delta-\delta)] \\
& I_{2}=I_{0}[1+\gamma \cos (\Delta)] \\
& I_{3}=I_{0}[1+\gamma \cos (\Delta+\delta)],
\end{aligned}
$$

where $I_{0}$ is the average intensity, $\gamma$ is the modulation factor, $\delta$ is the constant phase shift, and $\Delta$ is the phase to be determined. The phase calculated at each point in the interferogram is

$$
\Delta=\arctan \left[\frac{I_{3}-I_{1}}{I_{3}+I_{1}-2 I_{2}}\right] \tan \delta / 2
$$

$\tan \delta / 2$ is unity as the phase shift in our case is $90^{\circ}$. The phase ambiguity was removed by comparing the phase difference between the adjacent pixel. The objectshape from the phase data is obtained by calculating $\Delta h$ from Eq. (7). The measured values of the tilt angle and the illumination angle are fed into the program for the shape determination.

Random variation of the speckle pattern causes noise in the data points. Moreover, the decorrelation of the speckle pattern due to deformation and the low modulation of the intensity at a given pixel value imposes further limitations on the speckle method.

A median window is introduced in the integration process to smooth the data points between the subtracted speckle patterns and to reduce phase discontinuities caused by the correction method. Apart 


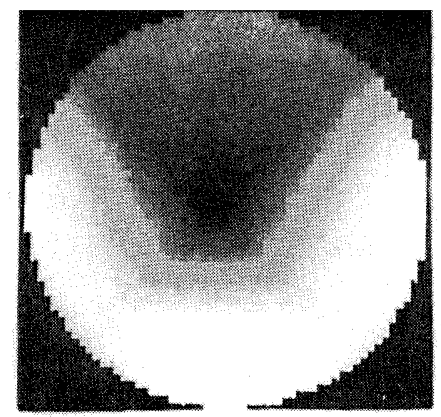

a

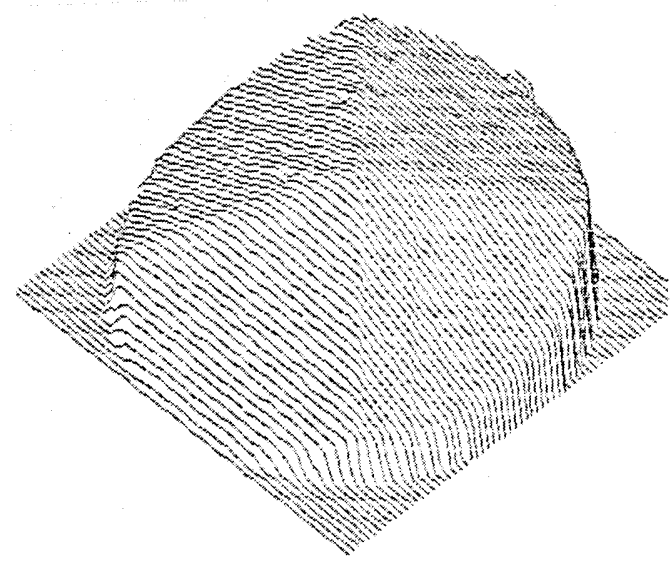

b

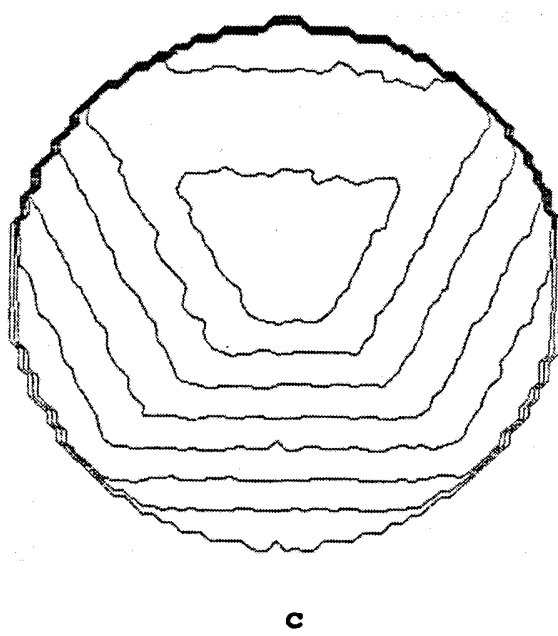

Fig. 5. Results when the contour planes are inclined. The interferogram shown in Fig. 3(c) was used here. a) Phase maps. b) 3-D plot. c) Contour maps.

from the smoothing of random data, there is an increase in the signal to noise ratio $(\mathrm{S} / \mathrm{N})$. This was determined by taking pixel values along a horizontal line cutting the fringes. The $\mathrm{S} / \mathrm{N}$ ratio was determined before and after the median window process from the Fourier transform of the pixel data. A plane object inclined by $10^{\circ}$ was chosen for the study and the fringes were perpendicular to the scanning of the data. $\mathrm{S} / \mathrm{N}$ without median windowing were found to be $<0.015$ for an optimum setting of the viewing system at $F / 10$. The $\mathrm{S} / \mathrm{N}$ was found to increase as the size of the window was increased, and also with the number of windows used sequentially. A $3 \times 3$ and $5 \times 5$ median window used sequentially to smooth the speckle pattern data was found to yield good results $(\mathrm{S} / \mathrm{N}=0.05)$.

The effect of decorrelation, which is an intrinsic property (this arises due to change in the effective rays collected by the system before and after tilt of the object), can be minimized by adopting a large angle of illumination. In this case the sensitivity of the method becomes higher, thus reducing the tilt angle needed to produce a definite number of fringes. Furthermore the fringe signal varies slowly with the $F$-number of the viewing system and fringes of tolerable contrast were obtained even for speckle smaller than the pixel size of the CCD camera. ${ }^{11}$ The optimum $\mathrm{S} / \mathrm{N}$ ratio in our case was obtained when the speckle size was of the order of the resolution of the camera, i.e., $20 \mu \mathrm{m}$. It was found experimentally that with a larger aperture satisfactory results with the fringe evaluation program were obtained.

\section{Results and Discussion}

The experimental arrangement used for contouring is shown in Fig. 1. A $15-\mathrm{mW} \mathrm{He}-\mathrm{Ne}$ laser beam is split into two illuminating beams by a beam splitter. A CCD camera with a Nikon objective was set to $F / 10$ by an external aperture. The speckle patterns were digitized on a Epson host computer and displayed on a TV monitor. The photographs were directly taken from the monitor. Figure 3(a) shows the photograph with the contour fringes for a pyramid with an apex angle of $120^{\circ}$. The contour planes lie parallel to the $x y$-plane, which is also the observation direction. In Fig. 3(b) the object is inclined about the $y$-axis by $10^{\circ}$, giving a different perspective view of the object. On the other hand Fig. 3(c) shows the fringes for an object where the contour planes are tilted about the $x z$-plane.

The fringe analysis with phase shifting was carried out for the interferogram shown in Fig. 3(a). $3 \times 3$ and 


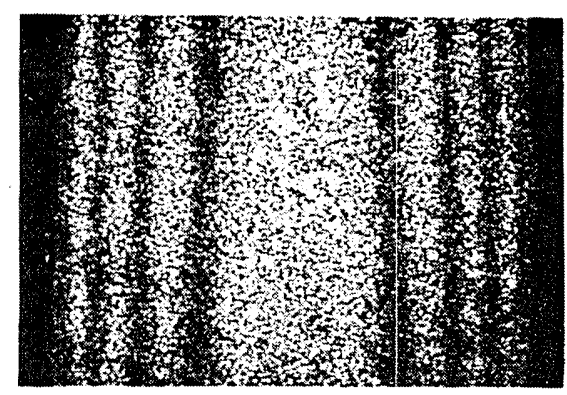

a

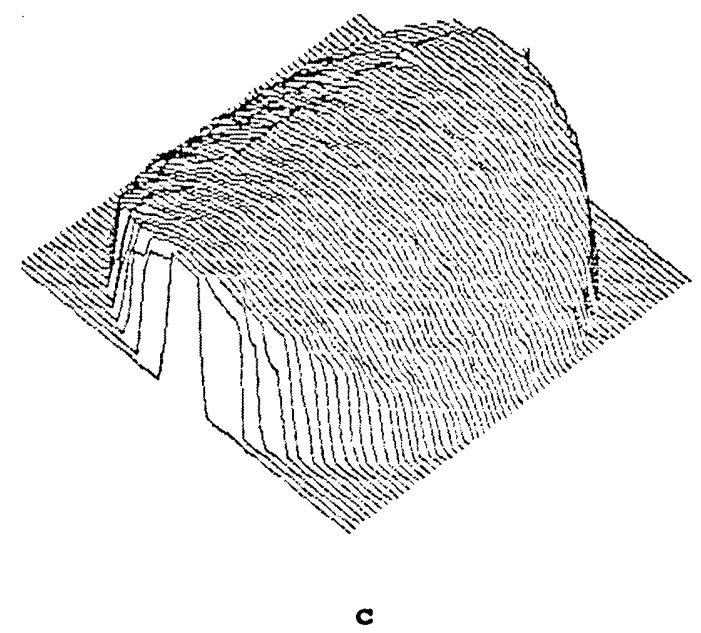

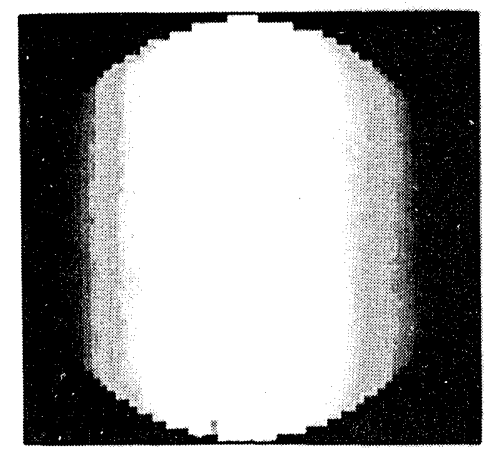

b

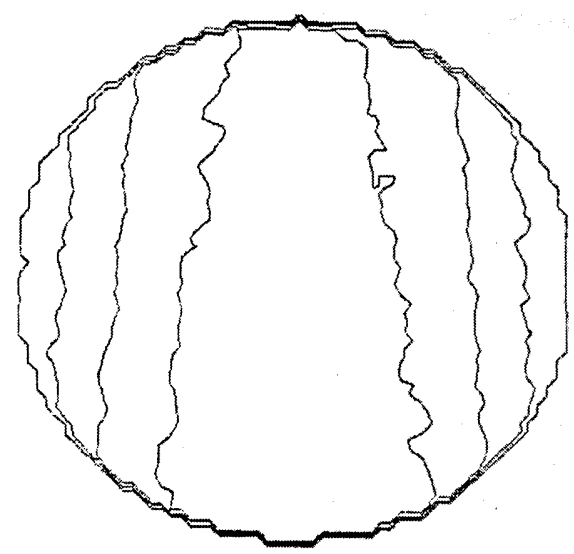

d

Fig. 6. Results for a cylindrical surface whose radius of curvature is $74.5 \mathrm{~mm}$ : a) Photograph of the contour fringes. b) Phase maps. c) 3-D plot. d) Contour maps. The data for this interferogram are: $\beta=0, \phi_{t}=0.55 \mathrm{mrad}, \theta=45^{\circ}$, and $\Delta h=0.81 \mathrm{~mm}$.

$5 \times 5$ median windows were used to evaluate the phase, and Figs. 4(a,b) and (c) show the phase, 3-D plot and the contour map respectively. Consistent results were obtained by employing two median window of $3 \times 3$ and $5 \times 5$ sequentially in the integration process. Figures $5(a, b)$ and $(c)$ are the results when tilt and rotation of the object were introduced to incline the contour planes. A final example of the method is demonstrated for a cylindrical surface and is shown in Figs. $6(a, b, c)$ and $(d)$. For the results demonstrated the objects were sprayed with matt white spray to obtain uniform scattering.

The experimental results show good agreement with the theoretical predictions. The results demonstrated with phase shifting, indicate the use of the dual beam illumination method as a tool for 3-D topography. In spite of the large illuminating angle, the setup was found to be quite stable. Although tilt was introduced mechanically in the experiment, with a stepping motor the tilt can be computer controlled. For these experiments no spatial filter was used. Furthermore, good results with fringe analysis were obtained. The arrangement can be made more compact by adopting a single large illuminating beam and a mirror at $90^{\circ}$ to the object, which has been used in speckle metrology. ${ }^{6}$ However, in such an arrangement the sensitivity of the method is fixed as the illuminating angle is fixed at $45^{\circ}$.

In conclusion, we have demonstrated a method for generating contours of an object. The method is simple and stable provided the initial conditions are met to eliminate the other two components of displacement. The phase contours of the object shape have been determined by phase shifting techniques. This allows the shape of the object to be measured accurately. The accuracy of the method depends on the accuracy of measuring the tilt angle. If the angles of the two illuminating beams are equal and the slope of the object under investigation is small, the visibility of the fringes can be shown to be dependent on the angle of illumination, tilt of the object, and the surface roughness of the object. A compromise between the tilt angle and the depth of the object can be obtained for a particular visibility. The contour fringes are not strickly dependent on the position of the tilt axis. Further this interferometer has also been used for: a) an indirect method of measuring the tilt of an object to a very high sensitivity, b) measurement of the refrac- 
tive index variation of a medium, c) testing of optical elements such as lenses and aspheric surfaces, d) comparison of two asperic surfaces, and e) measurement of rotation. These studies are in progress and results will be published later.

Charles Joenathan, an Alexander von Humboldt fellow, would like to thank the Alexander von Humboldt foundation for financial support.

\section{References}

1. K. A. Haines and Hildebrand, "Contour Generation by Wavefront Reconstruction," Phys. Lett. 19, 10-11 (1965).

2. J. S. Zelenka and J. R. Varner, "A New Method for Generating Depth Contours Holographically," Appl. Opt. 7, 2107-2110 (1968).

3. N. Abramson, "Sandwich Hologram Interferometry. 3: Contouring," Appl. Opt. 15, 200-205 (1976).

4. P. DeMattia and V. Fossati-Bellani, "Holographic Contouring by Displacing the Object and the Illuminating Beam," Opt. Commun. 26, 17-21 (1978).
5. J. N. Butters, R. C. Jones, and C. Wykes, "Electronic Speckle Pattern Interferometry," in Speckle Metrology, R. K. Erf, Ed. (Springer Verlag, city, 1975) pp. 111-157.

6. R. Jones and C. Wykes, Holographic and Speckle Interferometry (Cambridge U.P., London 1983).

7. B. D. Bergquist and P. Montgomery, "Contouring by Electronic Speckle Pattern Interferometry (ESPI),” Proc. Soc. Photo-Opt. Instrum. Eng. 599, 189-195 (1985).

8. A. R. Ganesan and R. S. Sirohi, "New Method of Contouring Using Digital Speckle Pattern Interferometry," Proc. Soc. Photo-Opt. Inst. Eng. 954, 327-332 (1988).

9. J. A. Leendertz, "Interferometric Displacement Measurement on Scattering Surfaces Utilizing Speckle Effect," J. Physics E. 3, 214-218 (1970).

10. Y. Y. Hung, "Displacement and Strain Measurement," in Speckle Metrology, R. K. Erf, Ed. (Springer Verlag, New York, 1975), pp. 51-71.

11. C. Wykes, "A Theoretical Approach to the Optimization of Electronic Speckle Interferometry with Limited Laser Power," J. Mod. Opt. 34, 539-554 (1987). 